\title{
Androgen Regulation of Muscle Fiber Type in the Sexually Dimorphic Larynx of Xenopus laevis
}

\author{
David A. Sassoon, ${ }^{a}$ Grace E. Gray, ${ }^{\mathrm{b}}$ and Darcy B. Kelley \\ Department of Biological Sciences, Columbia University, New York, New York 10027
}

We used histochemical techniques [assays for adenine triphosphatase (ATPase) and succinate dehydrogenase (SDHase) activity] to identify muscle fiber types in the larynx of Xenopus laevis. Male muscle is made up of one fiber type, medium-sized fibers $\left(\sim 9 \mu \mathrm{m}^{2}\right)$ that stain lightly for acid-stable ATPase and intensely for SDHase activity. In contrast, the female has 3 fiber types: small fibers $\left(\sim 6 \mu \mathrm{m}^{2}\right)$ that stain intensely for ATPase and SDHase, medium-sized fibers $\left(\sim 13 \mu \mathrm{m}^{2}\right)$ with moderate staining for ATPase and dark staining for SDHase, and large fibers $\left(\sim 15 \mu \mathrm{m}^{2}\right)$ with little SDHase or ATPase activity.

Long-term castration (6 months) has no effect on histochemical staining of adult male fibers. Long-term testosterone treatment (5 months) increases the proportion of medium-sized, moderately staining fibers in adult females, and reduces the proportion of both the small, darkly staining fibers and the large, lightly staining fibers. At metamorphosis, both males and females have 3 fiber types whose ATPase activity is similar to that of the adult female. However, no SDHase activity is observed. Treatment of juveniles for 3 weeks with testosterone results in nearly complete masculinization of muscle fibers, as judged by increased crosssectional area, homogeneous ATPase staining, and a marked increase in SDHase activity. Thus, juvenile muscle is considerably more responsive to testosterone than is adult female muscle. We propose that the uniform metabolic properties of male laryngeal muscle contribute to the production of the rapid $(66 \mathrm{~Hz}$ ) mate call vocalizations characteristic of this species. Further, our results suggest that androgens direct the masculinization of laryngeal muscle fibers during postmetamorphic development.

Many skeletal muscles involved in vertebrate reproductive behaviors exhibit a heightened sensitivity to androgens. Examples include the rat bulbocavernosus-levator ani complex (Cihak et al., 1970; Breedlove and Arnold, 1983a, b), the bird syrinx (Lieberburg and Nottebohm, 1979), the temporalis muscle of guinca pigs (Wainman and Shipounoff, 1941; Kochakian ct al.,

Received Dec. 29, 1986; accepted Apr. 1, 1987.

We wish to thank Dr. P. Nemeth for advice on muscle histochemistry and for a critical reading of the manuscript. Dr. C. Tountas helped with statistical methods and computer graphics. This work was supported by NIH Grant NS19949. D.B.K. was the recipient of NIH RCDA Award HD00493.

Correspondence should be addressed to Dr. D. B. Kelley, Dept. of Biol. Sci., 911 Fairchild, Columbia University, New York, NY 10027.

a Present address: Institut Pasteur, 28 rue du Dr. Roux, Cedex 15, Paris, France.

- Present address: Division of Biology and Biomedical Sciences, Washington University, St. Louis, MO 63108.

Copyright (C) 1987 Society for Neuroscience $0270-6474 / 87 / 103198-09 \$ 02.00 / 0$
1964), and the flexor carpi radialis of frogs (Thibert and Fardeau, 1978; Rubinstein et al., 1983). These specialized muscles have high levels of cytosolic androgen receptor (Jung and Baulieu, 1972; Lieberburg and Nottebohm, 1979; Max et al., 1981); muscle mass is larger in males (Wainman and Shipounoff, 1941; Venable, 1966; Thibert and Fardeau, 1978; Luine et al., 1980; Segil et al., 1987). Skeletal muscle fiber type and size have been shown to be influenced by steroid hormones (Venable, 1966; Muller et al., 1969; Cihak et al., 1970; Rubinstein et al., 1983; Lyons et al., 1986). In Xenopus laevis, for example, testosterone affects the number and distribution of tonic muscle fibers in the arm (Rubinstein et al., 1983).

We have been investigating the larynx of $X$. laevis, an androgen target, which serves as the effector organ for the male mate call (Wetzel and Kelley, 1983; Segil et al., 1987; Tobias and Kelley, 1987). At sexual maturity the adult male frog larynx is 3 times heavier than that of the female and has 8 times more muscle fibers, whereas at metamorphosis the larynges of both sexes have the female number of muscle fibers. During the first year following metamorphosis, laryngeal muscle fiber number increases from 4000 to 32,000 fibers in males (Sassoon and Kelley, 1986). Androgenic steroids induce myogenesis and chondrogenesis in the juvenile larynx (Sassoon et al., 1986). This proliferative response, observed after testosterone is administered to sexually immature frogs, contributes to the developmental program that results in a larger muscle mass and fiber number in adult males.

The larynx of adult male $X$. laevis produces prolonged bouts of calling by rapid and sustained contraction of the laryngeal dilator muscles. The female larynx cannot produce such rapid and sustained contractions either when examined in a behavioral context or when the laryngeal nerve is stimulated directly in vitro (Hannigan and Kelley, 1986; Tobias and Kelley, 1987). These data suggest that muscle fiber types may be different in adult male and female larynges. If so, we wished to determine how sex differences in fiber type arise during development and the extent to which androgenic steroids are involved in this process. In the present study we examine myosin-ATPase and mitochondrial-SDHase, using histochemical methods, in adult and juvenile laryngeal muscle. In addition, we assess androgen effects on staining patterns.

\section{Materials and Methods}

Animals and hormone treatment. Adult $X$. laevis were purchased from Nasco (Ft. Atkinson, WI) or Xenopus I (Ann Arbor, MI). Females were ovariectomized prior to hormone treatment (see Kelley et al., 1975, for operative procedures). Pellets of compressed crystalline testosterone propionate ( $30 \mathrm{mg}$; Sigma) were implanted through a small incision into the dorsal lymph sac and retained for 1 and 5 months. Males were 
castrated, which effectively removes circulating testosterone (Kelley, 1980), and laryngeal muscle was processed histochemically 6 months later. When killed, the animals were inspected to assure that the gonads had not regenerated. Juvenile animals were purchased from Nasco at metamorphic climax (stage 66; Nieuwkoop and Faber, 1956). The juveniles were treated for $1-5$ weeks with testosterone propionate pellets ( $3 \mathrm{mg}$ ) and compared to animals from the same ovulation group.

Tissue preparation. Frogs were killed while under deep anesthesia with $m$-amino benzoate methanesulfonic acid (MS-222; Aldrich). The larynges were dissected free of surrounding tissue and frozen in isopentane cooled with liquid nitrogen. Adult larynges were bisected along the midline and the medial cartilage cut away. The presence of some adjacent cartilage facilitated the orientation of the laryngeal muscles and also served to minimize muscle contraction artifact. Juvenile larynges were processed without prior dissection. The $\mathrm{m}$. semimembranosus muscle of the thigh was also dissected from each animal and processed for histochemistry. This muscle was used because it is not sexually dimorphic and, unlike the larynx, has only low levels of androgen binding (Segil et al., 1987), thereby allowing us to determine whether sex differences or hormone effects were specific to the laryngeal muscle.

Histochemistry. Tissue was cut perpendicular to the longitudinal axis of the muscle fibers at $-20^{\circ} \mathrm{C}$ in a cryostat (Bright Instrument Co.). Serial sections $(20 \mu \mathrm{m})$ from the midrostrocaudal portion of the larynx were placed on subbed slides and reacted for acid-stable adenine triphosphatase (ATPase) activity following a modification of the method of Guth and Samaha (1970). The acid preincubation ( $\mathrm{pH} \mathrm{4.6)} \mathrm{for} \mathrm{laryn-}$ geal muscle was determined in preliminary studies on thigh muscle to reliably yield differential staining of fiber types. Tissue sections adjacent to those used for the ATPase reactions were stained for succinate dehydrogenase (SDHase) activity using the technique of Nachlas et al. (1957). Adult tissue was incubated for $20 \mathrm{~min}$; longer time periods resulted in excessive crystallization of reaction product in the male larynx. Juvenile tissue was incubated for $2 \mathrm{hr}$, as little or no SDHase reactivity was apparent following shorter incubation periods. Frogs of both sexes and non-hormone-treated and hormone-treated animals were always reacted simultaneously to assure that any differences observed were due to sex or hormone treatment alone and not to histochemical reaction variability.

Morphometric analysis - fiber type determination. The maximal crosssectional area of laryngeal muscle occurs at the anterioposterior midpoint in both males and females (Sassoon and Kelley, 1986); consequently, this region was used for analysis. Photomicrographs were prepared using sections of laryngeal and $\mathrm{m}$. semimembranosus muscles. For each muscle fiber, staining for both acid-stable ATPase and SDHase activity was determined from photomicrographs of adjacent sections; assignments to fiber type were then made. In order to evaluate the differences observed in laryngeal muscle staining due to sex, age, and hormone trcatment, 3 animals from cach expcrimental group werc studied in detail. The determination of staining intensity for SDHase and ATPase was made on 150-200 fibers/muscle (i.e., 450-600 fibers were measured for each experimental group). In addition, muscle fiber crosssectional area for each fiber was obtained from photomicrographs using a MorphII bit pad (Morph II system; Peachey), allowing us to correlate fiber size with fiber-staining characteristics. Intensity of staining was quantified by light-transmittance readings of single fibers made directly from tissue sections using a camera lucida attached to a Zeiss microscope. The image of the section was projected at a final magnification of $10^{3} \times$. The amount of light transmitted through the fibers was measured with a photoamplifier attached to a digitized meter, and values were assigned to individual fibers identified from previously prepared photomicrographs. Three readings per fiber were averaged to compensate for the punctate nature of the SDHase reaction; rcadings through the nuclei of the muscle fibers were discarded. Relative absorbance (light absorbed + light reflected) was arrived at by subtracting the average transmittance value for each fiber from the transmittance value of unreacted tissue or tissue processed without enzyme substrate. This approach in different fibers and different muscles served to evaluate effects of age, sex, and hormone treatment and to facilitate comparison of staining patterns. Fiber sizes (mean cross-sectional area; see above) were presented as mean $( \pm$ SD). Both SDHase- and ATPase-reacted materials were examined. In adult males, relative light absorbance was essentially the same for all fiber sizes. In females, however, relative light-absorbance readings revealed a trimodal (dark, moderate, light) distribution of values. Fibers were thus assigned to one of these 3 categories, and fiber types (darkly, moderately, and lightly staining classes) were correlated to fiber size. Since adult female and juvenile fiber sizes are not normally distributed, the significance of differences in size between fiber populations was evaluated using the Median test (Siegel, 1956).

\section{Results}

Adults

Thigh. To assist in fiber typing, a thigh muscle, m. semimembranosus, was processed for comparison with laryngeal muscle. In both sexes, thigh muscle contains small fibers that are highly reactive for both acid-stable ATPase and SDHase (SD), medium-sized fibers that react moderately for ATPase and strongly to moderately for SDHase (MM), and larger fibers that react lightly for both SDHase and ATPase (LL) (see Fig. 1).

Larynx. Laryngeal muscle fibers (Fig. 2) are very small compared to $\mathrm{m}$. semimembranosus (Fig. 1). Adult male laryngeal muscle fibers have a median fiber cross-sectional area of $9 \mu \mathrm{m}^{2}$ (Fig. 3). In contrast, the femalc has a wider range of fiber crosssectional areas (Fig. 4). The majority of fibers in the female are smaller than those of the male; the median cross-sectional area in females is $6 \mu \mathrm{m}^{2}$.

The male laryngeal muscle consists of fibers that stain moderately for acid-stable ATPase (Fig. 2a). The SDHase reaction product in male larynx was extremely intense compared to that of the female (compare Fig. 2, $b$ and $d$ ). Staining for ATPase and SDHase was uniform among the fibers of males (Fig. 2, a, $b$ ), indicating only one fiber type. In females, however, both SDHase and ATPase reactions (Fig. 2, $c, d$ ) revealed a heterogeneous pattern of staining similar to that observed in thigh muscle.

To determine whether fiber size and fiber histochemistry are correlated, the cross-sectional areas of individual fibers of female laryngeal muscle were compared to fiber type. Correlation of ATPase fiber-staining density with fiber size in females reveals that the darkly staining fibers are generally the smallest and that the lightly staining fibers tend to be larger. The female larynx also contains a small population $(6 \%)$ of medium-sized fibers similar in ATPase staining to those of the predominant fiber type class in the male (compare Figs. $3 b$ and $4 c$ ). The SDHase reactions reveal a similar staining pattern (Fig. $2 d$ ). As in thigh, darkly staining acid-stable ATPase fibers stain intensely for SDHase and lightly staining acid-stable ATPase fibers stain more lightly for SDHase.

Effects of hormone treatment. Treatment of adult females for 1 month with testosterone resulted in an increase in laryngeal muscle mass. However, the pattern of SDHase and ATPase staining in laryngeal muscle was indistinguishable from that of untreated females (data not shown). After 5 months of continuous testosterone treatment, an overall increase in SDHase activity was evident, as was a change in the acid-stable ATPase staining pattern (compare Figs. 4 and 5). The proportion of darkly staining fibers decreases from 74 to $57 \%$ and that of lightly staining fibers decreases from 20 to $10 \%$. Fiber size is also affected by testosterone treatment; the median area of the fiber population increases from 6 to $13.5 \mu \mathrm{m}^{2}$. Only the darkly staining fibers exhibit a significant size change $\left(5.5-13 \mu \mathrm{m}^{2} ; p<\right.$ 0.001 ). While there are significant shifts in fibcr-staining characteristics, no androgen-treated female larynx exhibits the homogeneous staining pattern characteristic of the male.

Adult males castrated for 6 months show no change in staining pattern for either ATPase or SDHase activity. In no case do castrated males exhibit the heterogeneous staining pattern characteristic of the female larynx. 

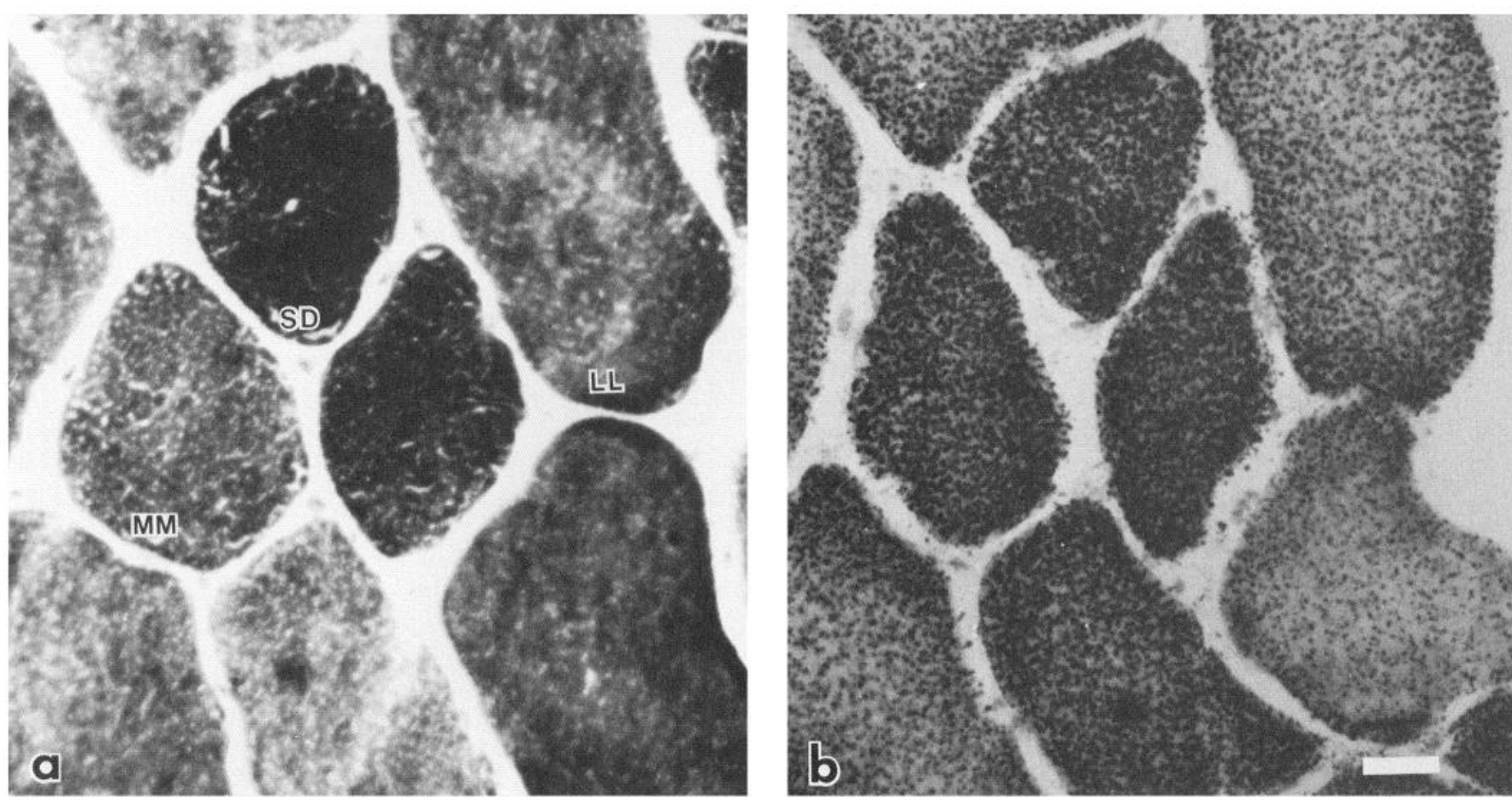

Figure 1. Photomicrographs of cross sections through adult thigh muscle, $\mathrm{m}$. semimembranosus. a, Male m. semimembranosus reacted for acidstable ATPase. $b$, Section adjacent to $a$ reacted for SDHase. Small, darkly staining $(S D)$, medium, moderately staining $(M M)$ and large, lightly $(L L)$ staining fibers can be distinguished. An identical staining pattern was obtained in females (not shown). Scale bar, $5 \mu \mathrm{m}$.

\section{Juveniles}

Fiber size and histochemistry. Morphological observations of stage 66 tadpoles reveal that the larynx is not yet sexually dimorphic in overall laryngeal shape, muscle and cartilage mass, cell number, or muscle fiber number (Sassoon and Kelley, 1986). Thus, we examined fiber types in male and female juveniles before the onset of sexual dimorphism, and androgen effects on muscle histochemistry. A single ovulation group of stage 66 animals (siblings) was used for this study. Half of the animals were treated with testosterone for 3-5 weeks before they were killed; the other half were untreated.

Histochemical analysis reveals a heterogeneously staining population of fibers in 3-5-week-old male and female postmetamorphic juvenile laryngeal muscle (Figs. 6-8). The ATPase staining pattern and distribution of juvenile muscle fiber types is similar to that found in adult females, although the muscle fibers are 3-4 times smaller in cross-sectional area (Figs. 7, 8). SDHase activity, however, is undetectable in juvenile larynx even after extending the incubation time from $20 \mathrm{~min}$ to $2 \mathrm{hr}$ (Fig. $6 b$ ). Thigh muscle staining is similar to that of adults, indicating that SDHase activity is present in other skeletal muscles at this time in development (data not shown).

In preliminary studies, stage 66 tadpoles were treated with testosterone for $1,1.5,2,3,4$, and 5 weeks. Although we could detect changes in fiber-staining patterns following a treatment as brief as 1 week, more dramatic results followed a 3-5 week hormone treatment period, and therefore animals treated for this time period were analyzed in detail. After 5 weeks of testosterone treatment, we observed in both sexes a homogeneous population of fibers staining moderately for ATPase and exhibiting moderately high SDHase activity (see Figs. 9, 10). A few, very small, darkly staining fibers for ATPase are also observed.
We do not observe any large, lightly staining fibers following hormone treatment in juveniles. The median fiber size found in 3-5 week testosterone-treated juveniles is comparable to the median size found in adult males (testosterone-treated juvenile male $=7.8 \mu \mathrm{m}^{2}$; testosterone-treated juvenile female $=9.2 \mu \mathrm{m}^{2}$; adult male $=9 \mu \mathrm{m}^{2}$ ). The $\mathrm{m}$. semimembranosus thigh muscle of both male and female juveniles was also examined; no obvious effects on staining patterns were detectable.

\section{Discussion}

\section{Fiber types in laryngeal muscle}

Vertebrate skeletal muscle consists of 2 major fiber types, which have been termed "tonic" and "twitch" (Burke, 1978). True tonic fibers are seldom broken down into subclasses. The "twitch" type fibers, however, have been classified into 2 subgroups: fasttwitch and slow-twitch. Although most efforts have been directed at mammalian skeletal muscle, this general classification scheme appears to apply also to amphibia (Tasaki and Mizutani, 1944; Kuffler and Gerard, 1947). Lannergren and Smith (1966) were able to classify muscle fibers in the m. iliofibularis of $X$. laevis on the basis of physiological properties and mitochondrial content. They confirmed that at least 2 types of twitch fibers exist: one group exhibits a short twitch time duration (fasttwitch) and has a relatively low mitochondrial content; a second group has a slightly longer twitch duration (slow-twitch) and a high mitochondrial content. In addition, they noted slow or nontwitch type fibers (tonic), which were "clear" when stained for mitochondrial content. Tonic fibers could be definitively distinguished from twitch type fibers, since they did not conduct propagating action potentials when stimulated. In addition, these authors showed that the mitochondrial content, as measured by enzymatic activity (SDHase and DPNase), was correlated with 

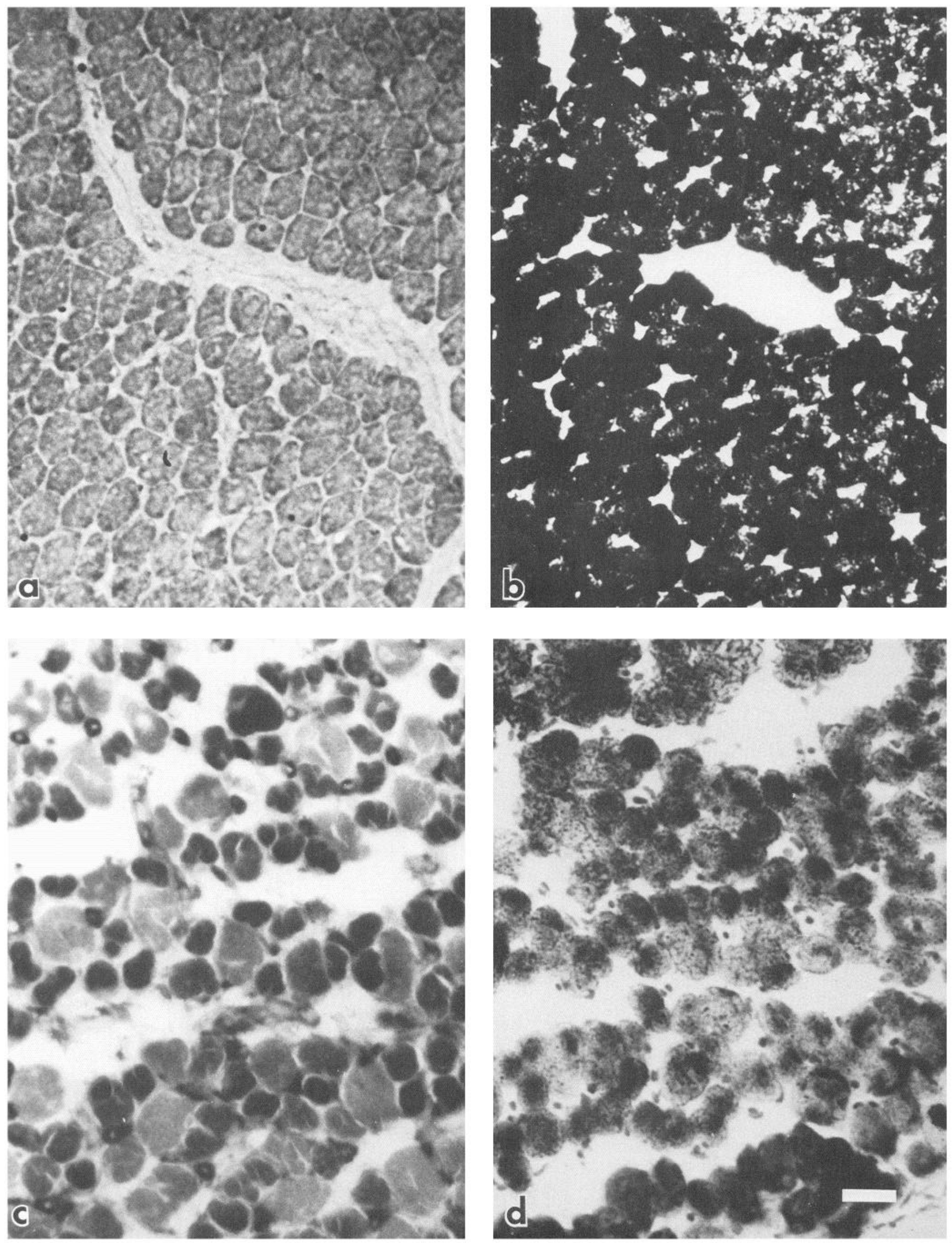

Figure 2. Photomicrographs of cross sections through adult laryngeal muscle, the bipinnate (dilator laryngis) at same magnification as in Figure 1. $a$, Male larynx reacted for acid-stable ATPase. $b$, Adjacent section reacted for SDHase. $c$, Female larynx reacted for acid-stable ATPase. $d$, Adjacent section reacted for SDHase. Scale bar, $5 \mu \mathrm{m}$. 


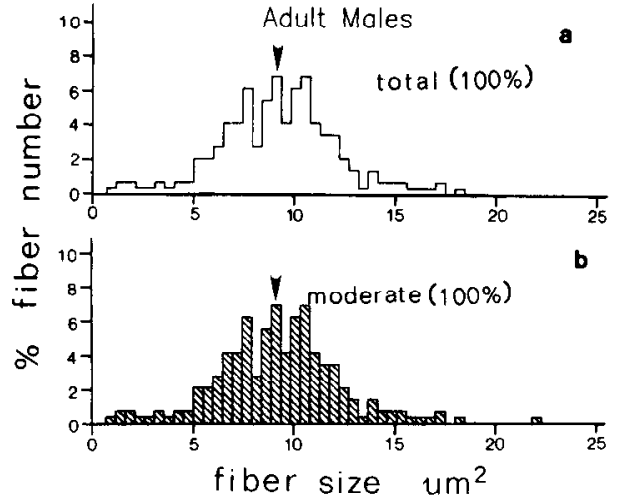

Figure 3. Histograms of laryngeal muscle fiber cross-sectional areas in the adult male. $a$, Total population of muscle fibers. $b$, Size distribution of fibers staining moderately for acid-stable ATPase. Arrowhead indicates median cross-sectional area. Three larynges were examined and at least 200 muscle fibers were identified from each individual.

the fatigue characteristics of the muscle fibers. Their findings were consistent with those of Peter et al. (1972) in mammalian fibers. High mitochondrial content is indicative of high oxidative capacity (Nachlas et al., 1957) and is associated with fatigue resistance, whereas low mitochondrial content and oxidative capacity are associated with rapid fatigue following frequent stimulation. Lannergren and Smith (1966) also found that fiber size and type could be correlated. In general, large fibers (100$150 \mu \mathrm{m}$ diameter) were fast-twitch and low in oxidative capacity, whereas small fibers (35-70 $\mu \mathrm{m}$ in diameter) were slowtwitch and high in oxidative capacity. Subsequent to Lannergren and Smith's (1966) work in X. laevis, histochemical methods were introduced that distinguished twitch-duration properties of mammalian muscle. Guth and Samaha (1970) demonstrated that myosin-ATPase enzymatic activity could be visualized histochemically. ATPase staining under different preincubation $\mathrm{pH}$ conditions has proved an aid in distinguishing fiber twitch type (Brooke and Kaiser, 1970; Burke, 1978).

A comparison of our results in the frog $\mathrm{m}$. semimembranosus muscle with those of Lannergren and Smith in $\mathrm{m}$. iliofibularis suggests that the $\mathrm{m}$. semimembranosus consists of at least 2 sorts of twitch fibers, large fibers that stain lightly for SDHase and small fibers that stain darkly for SDHase. If the twitch speed of the $\mathrm{m}$. semimembranosus is similar to that of the $\mathrm{m}$. iliofibularis (another thigh muscle in Xenopus), we would expect the large pale fibers to be fast-twitch fatigable and the small dark fibers to be slow-twitch fatigue-resistant. Our results with acidstable ATPase in the thigh are consistent with this prediction. We observe that the small fibers are darkly staining for acidstable ATPase (slow-twitch) and the large fibers are lightly staining for acid-stable ATPase (fast-twitch). There is also a fiber population that has moderate staining for both histochemical reactions. The fibers in this histochemical category are medium in size. This type of fiber was also observed by Lannergren and Smith (1966) in the $\mathrm{m}$. iliofibularis and was found to have moderate- to fast-twitch contraction properties with intermediate fatigue characteristics.

Laryngeal muscle fibers of $X$. laevis are very small compared to other frog skeletal muscle (i.e., $0.5-5.0 \mu \mathrm{m}$ in diameter, as compared to a $10-200 \mu \mathrm{m}$ diameter in $\mathrm{m}$. semimembranosus or m. iliofibularis (Lannergren and Smith, 1966)). The male larynx has a single population of medium-sized fibers with acid-

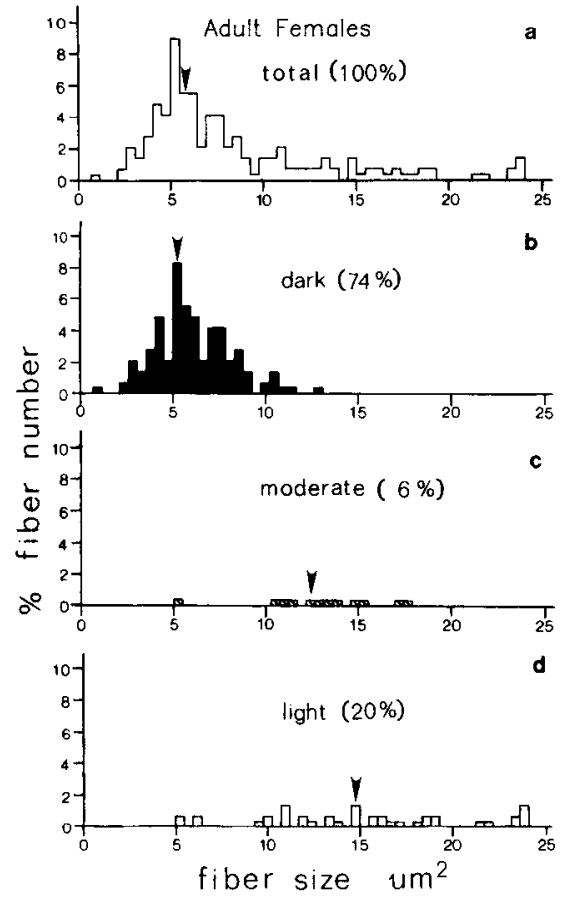

Figure 4. Histograms of laryngeal muscle fiber cross-sectional areas in the adult female. $a$, Total population of muscle fibers. $b-d$, Size distributions of fibers that have a dark, moderate, or light reaction product following histochemical reaction for acid-stable ATPase. $A r$ rowheads indicate median cross-sectional areas for each population. Three larynges were examined and at least 200 muscle fibers were identified from each individual.
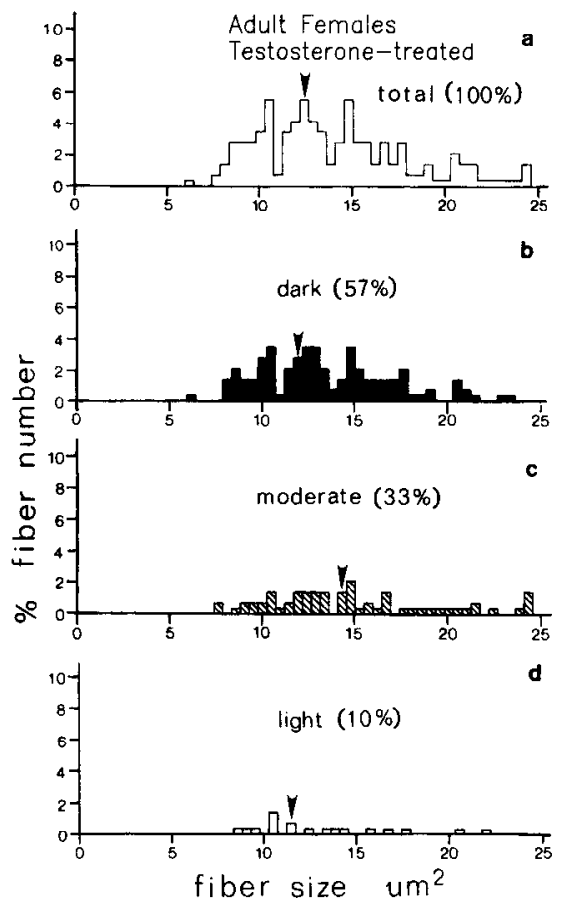

Figure 5. Histograms showing distribution of adult female laryngeal muscle cross-sectional areas following 5 months of testosterone treatment. $a$, Total population of muscle fibers. $b-d$, Three different acidstable ATPase staining classes of fibers correlated to fiber size. Arrowheads indicate median cross-sectional areas for each population. Two larynges were examined and at least 200 muscle fibers were identified from each individual. 

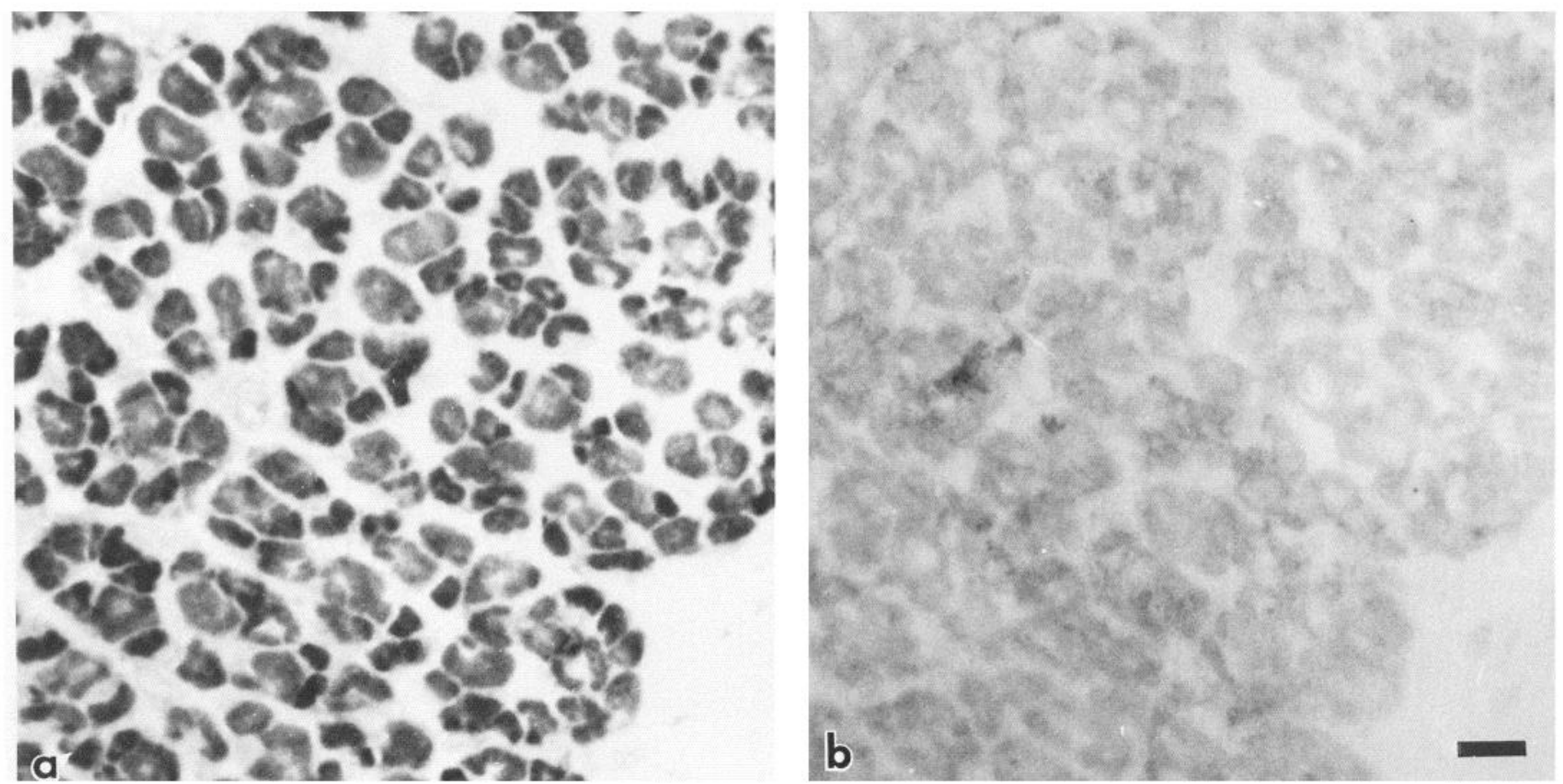

Figure 6. Photomicrographs of cross sections through a juvenile male laryngeal muscle. $a$, A section reacted for acid-stable ATPase. $b$, Adjacent section reacted for SDHase. An identical staining pattern was obtained in females (not shown). Scale bar, $2 \mu \mathrm{m}$.

stable ATPase activity. On the basis of the ATPase staining under acid conditions, we would predict an intermediate to light SDHase staining result. Instead, we observe uniformly intense SDHase reactivity throughout the muscle, indicating that male laryngeal muscle is intermediate- to fast-twitch and extremely oxidative. This combination of metabolic capacities suggests that male laryngeal muscle is made up of fibers capable of the prolonged bouts of rapid contractions required for male-specific vocalizations (Wetzel and Kelley, 1983). The male fiber type is similar to mammalian type IIa (Nemeth et al., 1979).

Female laryngeal muscle consists of a heterogeneous population of fibers resembling the heterogeneous fibers found in $\mathrm{m}$. semimembranosus. The most numerous fiber type is small in cross-sectional area, with high acid-stable ATPase and SDHase activity, and is likely to be a slow-twitch oxidative fiber type, i.e., mammalian fiber type I (Nemeth et al., 1979). A small population $(6 \%)$ of female laryngeal muscle fibers exhibits moderate acid-stable ATPase staining and moderate to high SDHase activity. This second class of fiber in the female resembles the one muscle fiber type observed in the male. A third population in the female larynx consists of larger fibers having low acidstable ATPase and SDHase activity. This fiber type is probably fast-twitch and more fatigable than the smaller fibers, and may represent a class similar to mammalian type IIb (Nemeth et al., 1979). We do not believe there are tonic fibers in laryngeal muscle; since ongoing studies using intracellular recordings have thus far failed to reveal fibers that do not conduct action potentials (Tobias and Kelley, 1986) and there are no fibers with "clear" staining for SDHase-a characteristic of tonic fibers (Lannergren and Smith, 1966).

\section{Hormone treatment and castration}

Androgen controls mate calling in adult males (Wetzel and Kelley, 1983). Behavioral studies on adult female frogs treated with testosterone for periods as long as 4 months reveal that female frogs cannot produce a malelike mate call (Hannigan and Kelley, 1986). One explanation for this lack of behavioral plasticity in the adult female may be that there are limitations of the laryngeal muscle in response to hormone. We observe that treatment of adult females with testosterone for 1 month does not induce any change in the overall pattern of acid-stable ATPase staining, although SDHase staining is increased slightly. Longer-term treatment (5 months) results in a 5-fold increase in the proportion of moderately staining medium-sized fibers (from 6 to $33 \%$ ). The moderately staining fibers are similar in size and staining characteristics to the only fiber class found in the male.

The maintained presence of darkly and lightly staining fibers in the androgen-treated female may limit the ability of the muscle to display malelike muscle contractions (Tobias and Kelley, 1987) or malelike behavior (Hannigan and Kelley, 1986). Androgen-treated female larynges are capable of responding to a more rapid train of nerve stimulation than non-hormone-treated larynges (Tobias and Kelley, 1987). We suggest that the medium-sized, moderately staining (malelike) fibers are essential for the performance of mate calling, and that the female, even after testosterone treatment, does not possess a sufficient number of these fibers for rapid vocalization.

The larynx is also dimorphic in muscle fiber number; males have approximately 32,000 fibers, while females have approximately 4000 fibers (Sassoon and Kelley, 1986). The sexual dimorphism in $X$. laevis laryngeal fiber number is even more striking when one considers that only $6 \%$ (240 fibers) of the female laryngeal muscle fibers are malelike, as compared to the 32,000 fibers in the male. A parallel study has demonstrated that the number of muscle fibers in the female does not change following prolonged testosterone treatment (Sassoon and Kelley, 1986). Androgen treatment of females thus results in a larynx containing 1200 malelike fibers (33\%), which may fall below 

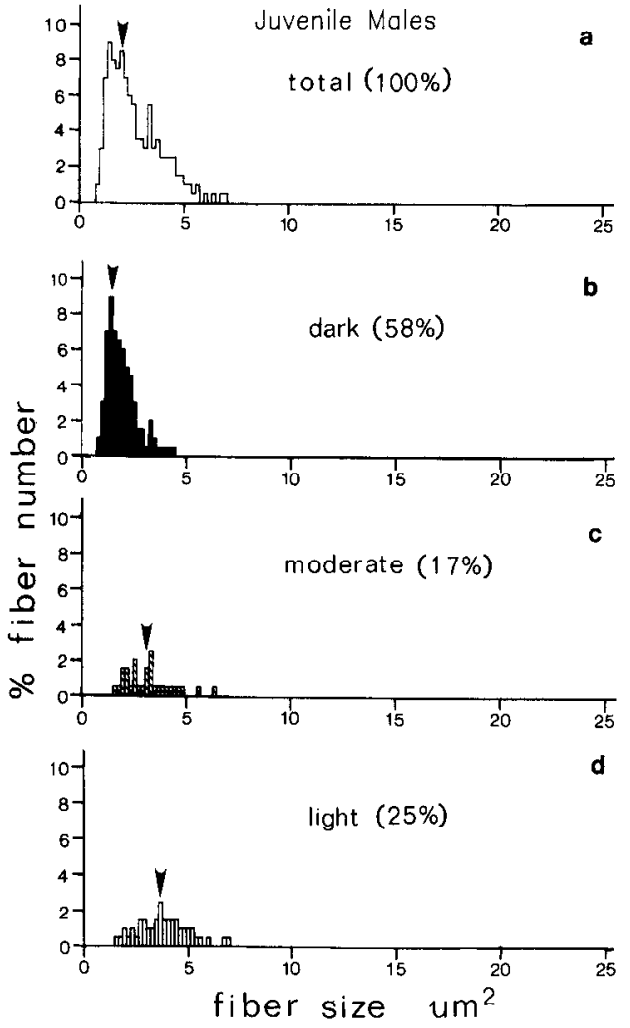

Figure 7. Histograms showing distribution of juvenile male laryngeal muscle fiber cross-sectional areas. $a$, Total population of muscle fibers. $b-d$, Three different ATPase staining classes of fibers correlated to fiber size. Arrowheads indicate median cross-sectional areas for each population. Three larynges were examined and at least 150 muscle fibers were identified from each individual.

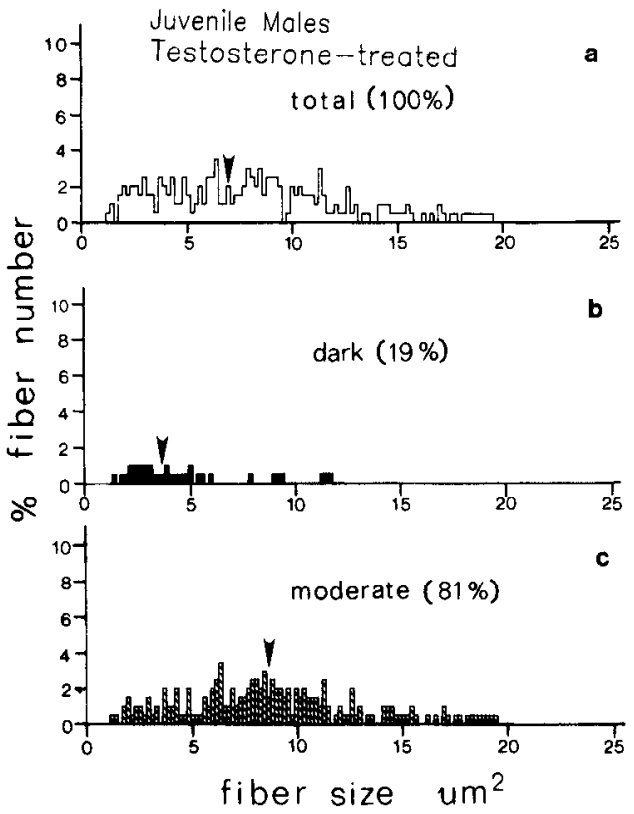

Figure 9. Histograms showing distribution of juvenilc malc laryngcal muscle fiber cross-sectional areas following 5 weeks of testosterone treatment. $a$, Total population of muscle fibers. $b, c$, Two different ATPase staining classes of fibers correlated to fiber size. Arrowheads indicate median cross-sectional areas for each population. Three larynges were examined and at least 150 muscle fibers were identified from each individual. The light-staining fiber class was not observed following 5 weeks of testosterone treatment.
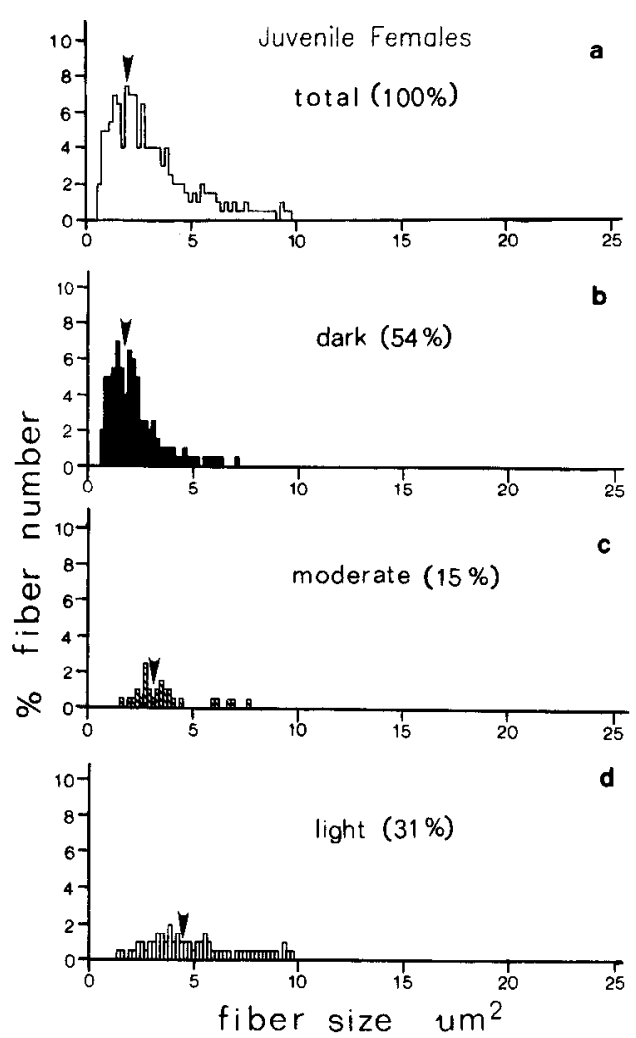

Figure 8. Histograms showing distribution of juvenile female laryngeal muscle fiber cross-sectional areas. $a$, Total population of muscle fibers. $b-d$, Three different ATPase staining classes of fibers correlated to fiber size. Arrowheads indicate median cross-sectional areas for each population. Three larynges were examincd and at lcast 150 muscle fibers were identified from each individual.
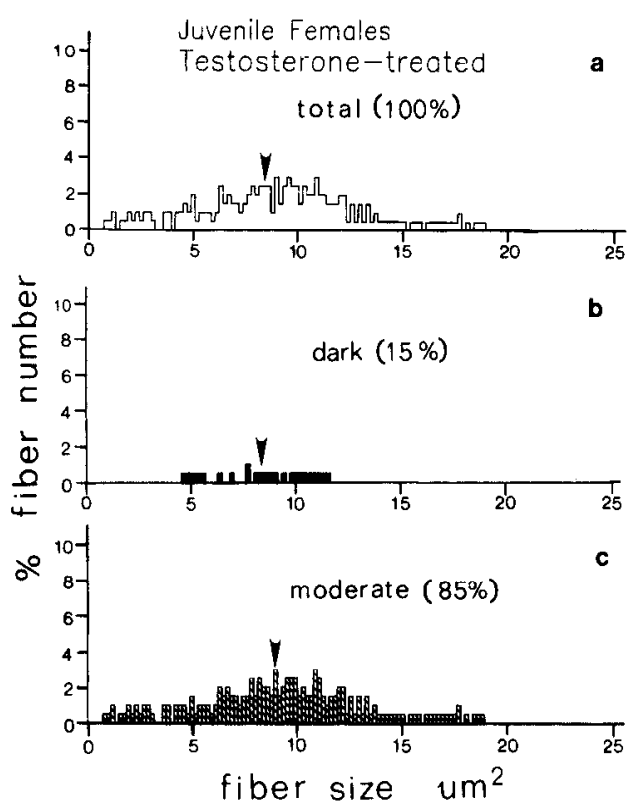

Figure 10. Histograms showing distribution of juvenile female laryngeal muscle fiber cross-sectional areas following 5 weeks of testosterone treatment. $a$, Total population of muscle fibers. $b, c$, Two different ATPase staining classes of fibers correlated to fiber size. Arrowheads indicate median cross-sectional areas for each population. Three larynges were examined and at least 150 muscle fibers were identified from each individual. The light-staining fiber class was not observed following 5 weeks of testosterone treatment. 
the critical number needed for masculine laryngeal function. We note that once fiber types are established in the adult male, lack of androgen (e.g., due to castration), even for a prolonged period, does not result in any obvious change in staining patterns in the male. Thus, the maintenance of the male fiber type is not hormone-dependent in adulthood.

\section{Juvenile larynges are sexually monomorphic}

The development of secondary sexual characteristics is controlled by hormonal factors (i.e., testosterone, Mullerian inhibiting substance) secreted by the developing testes (Wilson et al., 1981). These hormones act by modifying the body plan of the vertebrate embryo, which is essentially female (Jost, 1972; Wilson et al., 1981). Our findings on fiber types indicate that the larynx of $X$. laevis, like other secondary sex characteristics, is initially femalelike in muscle phenotype. Both male and female juvenile laryngeal muscle consists of 3 fiber types. The heterogeneity exhibited by both sexes in juveniles is similar to the staining observed in the adult female. Almost no SDHase activity is observed in juvenile larynx, indicating that mitochondrial content is low. In adulthood, females retain heterogeneity of fiber type, but males exhibit homogeneous staining (and high oxidative capacity). We thus suggest that the larynx develops by masculinization of an initially sexually monomorphic muscle. That this process involves a loss of 2 classes of fibers or their conversion to the malelike staining type is supported by our results with hormone-treated juveniles.

\section{Juvenile larynx response to testosterone}

Testosterone treatment of juveniles of both sexes results in the masculinization of histochemical staining. Compared to testosterone effects on adult females, the response of the juvenile to testosterone differs in 3 major respects: it is more rapid (e.g., 3 weeks rather than 6 months); more complete masculinization in the resultant staining pattern is observed following testosterone treatment; and the larger acid-stable ATPase light-staining fibers are no longer observed. One plausible explanation of these results is that the cellular mechanisms underlying fiber type expression in the larynx are different in adults and sexually immature animals. It may be that a critical "window" exists during development when testosterone can masculinize the larynx by promoting fiber type switching or selective fiber loss and replacement.

What accounts for the increased sensitivity and rapid response to testosterone observed in juveniles? A previous report from this laboratory has demonstrated that exogenously administered testosterone induces myogenesis in the larynx of sexually immature $X$. laevis, but not in adults or in other skeletal muscle (Sassoon et al., 1986). Since juveniles of either sex respond to testosterone by forming new muscle, it is possible that myogenesis contributes to the juvenile fiber type switching response. Adult laryngeal muscle contains androgen receptor levels that are higher than those observed in other skeletal muscle in the frog (Segil et al., 1987). We propose that elevated levels of receptor are prerequisite to the hormone sensitivity exhibited in juveniles. Recent studies from this laboratory have demonstrated that juvenile laryngeal tissue contains an order of magnitude more androgen receptor than that of adult males (Sassoon et al., 1985; Kelley et al., 1987). Thus, myogenic capacity and high levels of hormone receptor may contribute to the rapid change of fiber types in juveniles. The lower levels of receptor and attenuated proliferative response in the adult female could preclude complete and rapid fiber type switching.

The results presented here suggest that androgens can alter myosin type expression during development. It is not clear whether the hormonal effect is exerted at the level of the muscle or via nervous innervation. In birds, rats, and cats, fiber type can be switched (partially or completely) by changing the type of innervation (Karpati and Engel, 1967; Buller and Mommaerts, 1969; Weeds et al., 1974; Gauthier et al., 1983). During development, however, the initial pattern of fiber types is expressed appropriately by primary embryonic myotubes in the complete absence of innervation (Butler et al., 1982; Phillips and Bennett, 1984). The formation of secondary myotubes requires innervation (McLennan, 1983). Thus it is possible that androgen establishes the pattern of fiber types in the larynx by acting directly on the muscle during development, but maintains the metabolic state of the muscle fibers by regulating nerve activity in the adult. The former effect would be an "organizational" one; the latter would be "activational." In agreement with this view, we note that androgen eliminates the large, lightly staining fiber type in juveniles but not in adults.

The larynx in $X$. laevis is innervated by motor neurons that are sexually dimorphic in size and number (Hannigan and Kelley, 1981; Simpson et al., 1986). These motor neurons and their afferents also contain androgen receptor (Kelley et al., 1975; Kelley, 1980, 1981). It is unclear at present how these sex differences arise during development. Many central nervous system nuclei that participate in vocalization accumulate steroid hormones and thus could be regulated directly by receptorsteroid hormone action. Conversely, it is possible that the establishment of muscle fiber types influences motor neuron differentiation and survival. We hope that elucidation of the cellular processes involved in laryngeal muscle differentiation will help in the understanding of processes guiding the development of sex differences in the CNS.

\section{References}

Breedlove, S. M., and A. P. Arnold (1983a) Hormonal control of a developing neuromuscular system I. J. Neurosci. 3: 417-423.

Breedlove, S. M., and A. P. Arnold (1983b) Hormonal control of a developing neuromuscular system II. J. Neurosci. 3: 424-432.

Brooke, M. H., and K. K. Kaiser (1970) Three "myosin ATPase" systems: The nature of their $\mathrm{pH}$ lability and sulfhydryl dependence. J. Histochem. Cytochem. 18: 670-672.

Buller, A. J., and W. F. H. M. Mommaerts (1969) Myofibrillar ATPase as determining factor for contraction velocity, and its changes upon experimental cross-innervation. J. Physiol. (Lond.) 201: 46-47.

Burke, R. E. (1978) Motor units: Physiological/histochemical profiles, neuronal connectivity and functional specializations. Am. Zool. 18: 127-134.

Butler, J., E. Cosmos, and J. Brierley (1982) Differentiation of muscle fiber types in aneurogenic branchial muscles of the chick embryo. J. Exp. Zool. 224: 65-80.

Cihak, R., E. Gutmann, and V. Hanzlikova (1970) Involution and hormone-induced persistence of the $M$. sphincter levator ani in female rats. J. Anat. 106: 93-110.

Gauthier, G. F., R. E. Burke, S. Lowey, and A. W. Hobbs (1983) Myosin isozymes in normal and cross-reinnervated cat skeletal muscle fibers. J. Cell Biol. 97: 756-771.

Guth, L., and F. J. Samaha (1970) Procedure for histochemical demonstrations of actomyosin ATPase. Exp. Neurol. 28: 365-367.

Hannigan, P., and D. Kelley (1981) Male and female laryngeal motor neurons in Xenopus laevis. Soc. Neurosci. Abstr. 7: 269.

Hannigan, P., and D. Kelley (1986) Androgen-induced alterations in vocalizations of female Xenopus laevis: Modifiability and constraints. J. Comp. Physiol. A 158: 517-527. 
Jost, A. (1972) A new look at the mechanisms controlling sex-differentiation in mammals. Johns Hopkins Med. J. 130: 38-53.

Jung, I., and E.-E. Baulieu (1972) Testosterone "cytosol" receptor in the rat levator ani muscle. Nature (New Biol.) 237: 24-26.

Karpati, G., and W. K. Engel (1967) Transformation of the histochemical profile of skeletal muscle by "foreign" innervation. Nature 215: 1509-1510.

Kelley, D. (1980) Auditory and vocal nuclei of the frog brain concentrate sex hormones. Science 207: 553-555.

Kelley, D. (1981) Locations of androgen-concentrating cells in the brain of Xenopus laevis: Autoradiography with [3H]-dihydrotestosterone. J. Comp. Neurol. 199: 221-231.

Kelley, D., J. Morrell, and D. Pfaff (1975) Autoradiographic localization of hormone-concentrating cells in the brain of an amphibian, Xenopus laevis I. Testosterone. J. Comp. Neurol. 164: 47-62.

Kelley, D., D. Sassoon, and N. Segil (1987) Development and hormone regulation of androgen receptor levels in the sexually dimorphic larynx of Xenopus laevis. J. Cell Biochem. (Suppl.) 11A: 132.

Kochakian, C. D., J. Hill, and D. G. Harrison (1964) Regulation of nucleic acids of muscles and accessory sex-organs of guinea pigs by androgens. Endocrinology 74: 635-642.

Kuffler, S. W., and R. W. Gerard (1947) The small-nerve motor system to skeletal muscle. J. Neurophysiol. 10: 383-393.

Lannergren, J., and R. S. Smith (1966) Types of muscle fibers in toad skeletal muscle. Acta Physiol. Scand. 68: 263-274.

Lieberburg, I., and F. Nottebohm (1979) High-affinity androgen binding proteins in syringeal tissue of song birds. Gen. Comp. Endocrinol. 37: 286-293.

Luine, V., F. Nottebohm, C. Harding, and B. McEwen (1980) Androgen affects cholinergic enzymes in syringeal motorneurons and muscle. Brain Res. 192: 89-107.

Lyons, G., A. Kelly, and N. Rubinstein (1986) Testosterone-induced changes in contractile protein isoforms in the sexually dimorphic temporalis muscle of the guinea pig. J. Biol. Chem. 26: 13278-13284.

Max, S., S. Mufti, and B. Carlson (1981) Cytosolic androgen receptor in regenerating rat levator ani muscle. Biochem. J. 200:77-82.

McLennan, I. M. (1983) Neural dependence and independence of myotube production in chicken hindlimb muscles. Dev. Biol. 97: 229283.

Muller, E. R. A., G. Galavazi, and J. A. Szirmai (1969) Effect of castration and testosterone treatment on fiber width of the flexor carpi radialis muscle in the male frog (Rana temporaria L.). Gen. Comp. Endocrinol. 13: 275-284.

Nachlas, M. M., K. E. Tsou, E. Souza, C. S. Cheng, and A. M. Seligman (1957) Cytochemical demonstration of succinic dehydrogenase by the use of a new $p$-nitrophenyl substituted ditetrazole. J. Histochem. Cytochem. 5: 420-436.

Nemeth, P., H.-W. Hoefer, and D. Pette (1979) Metabolic heterogeneity of muscle fibers classified by myosin ATPase. Histochemistry 63: 191-201.

Nieuwkoop, P. D., and J. Faber (1956) Normal Table of Xenopus laevis (Daudin), North-Holland, Amsterdam.
Peter, J. B., R. J. Barnard, V. R. Edgerton, C. A. Gillespie, and K. E. Stempel (1972) Metabolic profiles of three fibre types of skeletal muscle in guinea pigs and rabbits. Biochemistry 11: 2627-2633.

Phillips, W. D., and M. R. Bennett (1984) Differentiation of fiber types in wing muscles during embryonic development: Effect of neural tube removal. Dev. Biol. 106: 457-468.

Rubinstein, N. $\Lambda$., S. D. Erulkar, and G. T. Schneider (1983) Sexual dimorphism in the fibers of a clasp muscle of Xenopus laevis. Exp. Neurol. 82: 424-431.

Sassoon, D., and D. Kelley (1986) The sexually dimorphic larynx of Xenopus laevis: Development and androgen regulation. Am. J. Anat. 177: 457-472.

Sassoon, D., N. Segil, and D. Kelley (1985) Development of sexual dimorphism in androgen receptor levels in the vocal organ of Xenopus laevis. J. Cell Biol. 101: 348a.

Sassoon, D., N. Segil, and D. Kelley (1986) Androgen-induced myogenesis and chondrogenesis in the larynx of Xenopus laevis. Dev. Biol. 113: 135-140.

Segil, N., L. Silverman, and D. Kelley (1987) Androgen binding in a sexually dimorphic muscle of Xenopus laevis. Gen. Comp. Endocrinol. 66: 95-101.

Siegel, S. (1956) Non-Parametric Statistics, McGraw-Hill, New York.

Simpson, H., M. Tobias, and D. Kelley (1986) Origin and identification of fibers in the cranial nerve IX-X complex of Xenopus laevis: Lucifer yellow backfills in vitro. J. Comp. Neurol. 244: 430-444.

Tasaki, I., and K. Mizutani (1944) Comparative studies on the activities of the muscle evoked by two kinds of motor nerve fibers. Jpn. J. Med. Sci. Biophys. 10: 237-244.

Thibert, P., and M. Fardeau (1978) Caracterisation histochemique des fibres toniques dans le muscle flexor carpi radialis de la grenouille (Rana temporaria L.). C. R. Acad. Sci. D (Paris) 286: 101-103.

Tobias, M., and D. Kelley (1986) Dye-coupling and physiology are sex-specific properties of laryngeal muscle fibers in the frog Xenopus laevis. Soc. Neurosci. Abstr. 12: 1213.

Tobias, M. L., and D. B. Kelley (1987) Vocalizations by a sexually dimorphic isolated larynx: Peripheral constraints on behavioral expression. J. Neurosci. 7: 3191-3197.

Venable, J. H. (1966) Morphology of the cells of normal, testosterone deprived and testosterone stimulated levator ani muscles. Am. J. Anat. 112: 271-302.

Wainman, P., and G. C. Shipounoff (1941) The effects of castration and testosterone propionate on the striated perineal musculature in the rat. Endocrinology 29: 975-978.

Weeds, A. G., D. R. Trentham, C. J. C. Kean, and A. J. Buller (1974) Myosin from cross reinnervated cat muscles. Nature 247: 135-139.

Wetzel, D. M., and D. B. Kelley (1983) Androgen and gonadotropin effects on male mate calls in South African clawed frogs, Xenopus laevis. Horm. Behav. 17: 388-404.

Wilson, J., F. George, and J. Griffin (1981) The hormonal control of sexual development. Science 211: 1278-1284. 